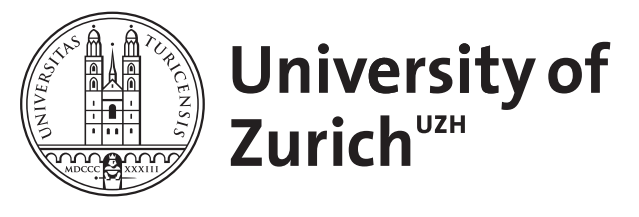

\title{
The first confirmed microlens in a globular cluster
}

\author{
Pietrukowicz, P ; Minniti, D ; Jetzer, P ; Alonso-García, J ; Udalski, A
}

\begin{abstract}
In 2000 July/August a microlensing event occurred at a distance of 2.33 arcmin from the center of the globular cluster M22 (NGC 6656), observed against the dense stellar field of the Milky Way bulge. We have used the adaptive optics system NACO at the ESO Very Large Telescope to resolve the two objects that participated in the event: the lens and the source. The position of the objects measured in 2011 July is in agreement with the observed relative proper motion of M22 with respect to the background bulge stars. Based on the brightness of the microlens components we find that the source is a solar-type star located at a distance of $6.0 \pm 1.5 \mathrm{kpc}$ in the bulge, while the lens is a $0.18 \pm 0.01$ $\mathrm{M}$ sun dwarf member of the globular cluster located at the known distance of $3.2 \pm 0.2 \mathrm{kpc}$ from the Sun. Based on observations collected with the ESO VLT and VISTA telescopes at Paranal Observatory (ESO Programmes 087.C-0640(A) and 179.B-2002(B), respectively), and the $1.3 \mathrm{~m}$ Warsaw telescope at the Las Campanas Observatory of the Carnegie Institution for Science.
\end{abstract}

DOI: https://doi.org/10.1088/2041-8205/744/2/L18

Posted at the Zurich Open Repository and Archive, University of Zurich

ZORA URL: https://doi.org/10.5167/uzh-70231

Journal Article

Published Version

Originally published at:

Pietrukowicz, P; Minniti, D; Jetzer, P; Alonso-García, J; Udalski, A (2012). The first confirmed microlens in a globular cluster. Astrophysical Journal Letters, 744(2):L18.

DOI: https://doi.org/10.1088/2041-8205/744/2/L18 


\title{
THE FIRST CONFIRMED MICROLENS IN A GLOBULAR CLUSTER*
}

\author{
P. Pietrukowicz ${ }^{1}$, D. Minniti ${ }^{2,3,4}$, Ph. Jetzer ${ }^{5}$, J. Alonso-García ${ }^{2}$, And A. Udalski ${ }^{1}$ \\ ${ }^{1}$ Warsaw University Observatory, Al. Ujazdowskie 4, 00-478 Warszawa, Poland \\ ${ }^{2}$ Departamento de Astronomía y Astrofísica, Pontificia Universidad Católica de Chile, Av. Vicuña MacKenna 4860, Casilla 306, Santiago 22, Chile \\ ${ }^{3}$ Vatican Observatory, Vatican City State V-00120, Italy \\ ${ }^{4}$ Department of Astrophysical Sciences, Princeton University, Princeton, NJ 08544-1001, USA \\ ${ }^{5}$ Institut für Theoretische Physik, Universität Zürich, Winterthurerstrasse 190, CH-8057 Zürich, Switzerland \\ Received 2011 November 10; accepted 2011 November 30; published 2011 December 15
}

\begin{abstract}
In 2000 July/August a microlensing event occurred at a distance of 2'.33 from the center of the globular cluster M22 (NGC 6656), observed against the dense stellar field of the Milky Way bulge. We have used the adaptive optics system NACO at the ESO Very Large Telescope to resolve the two objects that participated in the event: the lens and the source. The position of the objects measured in 2011 July is in agreement with the observed relative proper motion of M22 with respect to the background bulge stars. Based on the brightness of the microlens components we find that the source is a solar-type star located at a distance of $6.0 \pm 1.5 \mathrm{kpc}$ in the bulge, while the lens is a $0.18 \pm 0.01 M_{\odot}$ dwarf member of the globular cluster located at the known distance of $3.2 \pm 0.2 \mathrm{kpc}$ from the Sun.
\end{abstract}

Key words: globular clusters: individual (M22) - gravitational lensing: micro - instrumentation: adaptive optics

\section{INTRODUCTION}

The effect of gravitational microlensing of background stars by compact objects located in globular clusters was analyzed for the first time by Paczyński (1994). He showed that thanks to the usually well-known distances to the source and the lens, and transverse velocity between the populations to which the objects belong, it is possible to derive the lens mass when the event timescale is measured. Paczyński suggested monitoring globular clusters like M22 or 47 Tuc in front of the rich background of either the Galactic bulge or the Small Magellanic Cloud. According to his calculations one should detect up to a few microlensing events in one year of continuous monitoring of M22 with a $1 \mathrm{~m}$ class ground-based telescope. Some microlensing events detected so far toward the bulge in microlensing surveys such as OGLE (Udalski et al. 2000), MACHO (Alcock et al. 2000), and MOA (Bond et al. 2001) might be associated with globular clusters (Jetzer et al. 1998; de Luca \& Jetzer 2008).

Pietrukowicz et al. (2005) presented the results of a search for erupting objects in the field of the globular cluster M22. The cluster was observed over 10 weeks in 2000-2001 with the $1.0 \mathrm{~m}$ Swope telescope at Las Campanas Observatory as one of the targets of the Cluster AgeS Experiment (CASE; Kaluzny et al. 2005). Besides two erupting dwarf novae they found a probable microlensing event located at $\alpha_{2000.0}=18: 36: 22.40$, $\delta_{2000.0}=-23: 56: 29.4$, i.e., only $2 ! 33=1.75 r_{\mathrm{c}}=0.69 r_{\mathrm{h}}$ from the cluster center, where $r_{\mathrm{c}}=1$ '.33 and $r_{\mathrm{h}}=3$ '.36 are the core radius and half-mass radius of the cluster, respectively (taken from the 2010 version of Harris 1996 catalog). The brightness of the object increased by about 0.8 mag in $V$ over 20 days. Around 2000 August 5 it reached a maximum of $V=19.1 \mathrm{mag}$ and then faded to a constant level of $V=19.9$ mag. Based on its color at maximum brightness the authors excluded the possibility that the object could be a dwarf nova. They fitted a single lens model to the light curve and found that the most likely

\footnotetext{
* Based on observations collected with the ESO VLT and VISTA telescopes at Paranal Observatory (ESO Programmes 087.C-0640(A) and 179.B-2002(B), respectively), and the $1.3 \mathrm{~m}$ Warsaw telescope at the Las Campanas Observatory of the Carnegie Institution for Science.
}

geometry of the event places the source in the Galactic bulge and the lens in the cluster. The fitted parameters are: the epoch of maximum $t_{0}=2451759.70_{-0.34}^{+0.33}$, characteristic (Einstein) time $t_{\mathrm{E}}=15.9 \pm 1.1$ days, impact parameter $u_{0}=0.54_{-0.18}^{+0.02}$ in units of Einstein radius $r_{\mathrm{E}}, V_{\mathrm{S}}=19.92_{-0.02}^{+0.62} \mathrm{mag}$, and $V_{\mathrm{L}}=24.8_{-4.0}^{+\infty} \mathrm{mag}$, where $V_{\mathrm{S}}$ and $V_{\mathrm{L}}$ are the $V$-band magnitudes of the source and lens, respectively. The authors assessed the mass of the lens to $M=0.14_{-0.02}^{+0.10} M_{\odot}$. Large uncertainties of the above values result from the faintness of the object and partial coverage of the event.

After many years, in some special cases it is possible to directly detect the lens, measuring its mass and the geometry of the microlensing event (e.g., Alcock et al. 2001; Kozłowski et al. 2007). In this Letter, we resolve the microlensing system components based on new near-IR high-resolution images, measuring the complete geometry of the event and the parameters of the source and lens stars. The event reported here is the first confirmed microlensing event in a globular cluster. We note that brightening episodes detected in Hubble Space Telescope (HST) images of M22 by Sahu et al. (2001) were later re-examined and interpreted either as a dwarf nova type outburst (Anderson et al. 2003) or as a result of cosmic-ray double hits (Sahu et al. 2002).

\section{VLT OBSERVATIONS AND REDUCTIONS}

$K_{s}$-band observations of the M22-microlens region were obtained at the ESO Very Large Telescope (VLT) on 2011 July 17, i.e., 10.95 years after the maximum of the event. Twenty single $110 \mathrm{~s}$ exposures were taken using NACO at UT4, composed of the Nasmyth Adaptive Optics System (NAOS) and the High Resolution IR Camera and Spectrometer (CONICA). The detector was a $1026 \times 1024$ pixel SBRC InSb Alladin 3 array. We used the S27 camera of the scale 27.15 mas pixel $^{-1}$ and the field of view of $28^{\prime \prime} \times 28^{\prime \prime}$. The telescope jittered after each exposure according to a random pattern in an $8^{\prime \prime} \times 8^{\prime \prime}$ box. As the guide source for adaptive optics (AO) image correction we used a $V=14.1$ mag star located $11^{\prime \prime} .36$ away from the target. The seeing during the observations ranged between 0.'69 and 0.'99. The data were reduced with the ESO software packages MIDAS and Eclipse. In the top panel of Figure 1 we show the combined 


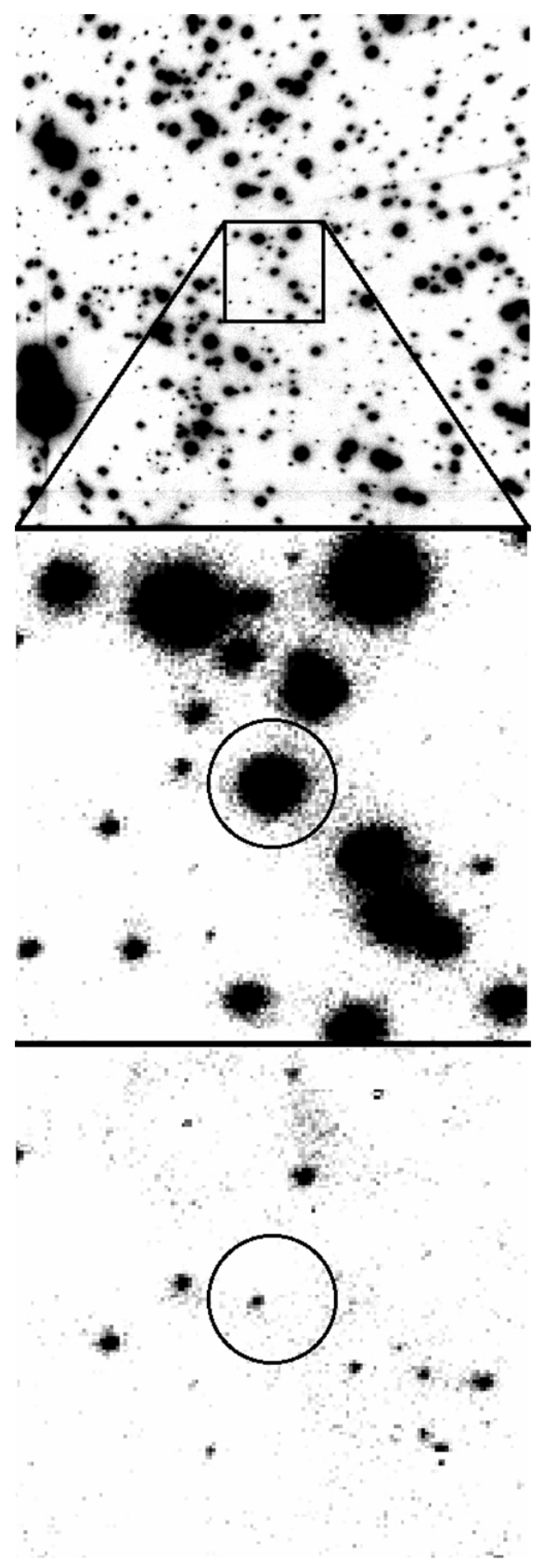

Figure 1. $K_{s}$-band images of the microlens in M22. The field of view in the top panel is $20^{\prime \prime}$ on a side. North is up and east is to the left. The brightest star near the SE corner of the top image served as the natural guide source for the AO image correction. The $4^{\prime \prime} \times 4^{\prime \prime}$ close-up view centered on the target source is presented in the middle panel. The lower panel shows a residual image after subtracting bright stars. The faint residual object located slightly off center is the lensing star.

image of the observed field. The image is affected by anisoplanatism, which degrades the point-spread function (PSF) making it more elongated with increasing angular distance from the guide star. The measured full width at half-maximum (FWHM) at the center of the image is $0{ }^{\prime \prime} 11$. The M22-microlens region was also observed with VLT/NACO through the $J$ filter on
2011 April 26. Unfortunately, the measured FWHM of 0.'36 is insufficient to detect the faint lens.

For our analysis we cut a smaller area of $600 \times 600$ pixels, covering $16^{\prime \prime} .3 \times 16^{\prime \prime} .3$ around the target microlens. We used DAOPHOT/ALLSTAR (Stetson 1987) to extract photometry of stars in the image. Due to relatively large difference in brightness $\left(\Delta K_{s}=3.2 \mathrm{mag}\right)$ and very small separation between the source and the lens ( 4.59 pixels $=124.6$ mas) the photometry was extracted in three steps. In the first step we found PSF based on selected isolated bright stars. Then using this PSF we found centroids of all stars with $\mathrm{S} / \mathrm{N}>3.5$. In the second step, we removed the bright stars from the image and extracted profile photometry for residual objects, including the lens. The residual image showing the lens located slightly off the center is presented in the lower panel of Figure 1. In the final step, we re-extracted the photometry for all stars including the positions of both the source and lens.

We performed simulations in which we inserted the same pair of stars in the same location of 100 frames with subtracted stars in order to assess the errors of the positions of the two objects. We converted the obtained mean uncertainties in pixels into units of mas.

Standard $K_{s}$-band magnitudes of the stars within our field were calculated based on photometry of 51 neighboring stars detected in the near-IR VISTA Variables in the Via Lactea survey (VVV; Minniti et al. 2010). We found the source and lens to have $K_{s}=17.37 \pm 0.03 \mathrm{mag}$ and $K_{s}=20.57 \pm 0.09 \mathrm{mag}$, respectively.

\section{CONFIRMATION OF THE MICROLENSING EVENT}

Almost eleven (10.95) years after the microlensing event we found the lens located $(123.6 \pm 1.8,15.8 \pm 1.8)$ mas (east, south) from the source. This corresponds to a relative proper motion of the lens with respect to the source $\left[\mu_{\alpha} \cos \delta, \mu_{\delta}\right]=$ $[11.29 \pm 0.17,-1.44 \pm 0.17] \mathrm{mas} \mathrm{yr}^{-1}$ and its total value $\mu_{\text {rel }}=$ $11.38 \pm 0.24$ mas $\mathrm{yr}^{-1}$. Based on archival HST observations Chen et al. (2004) measured the proper motion of the globular cluster M22 with respect to the background bulge stars. They obtained $\left[\mu_{\alpha} \cos \delta, \mu_{\delta}\right]=[10.19 \pm 0.20,-3.34 \pm 0.10]$ mas $_{\mathrm{yr}^{-1}}$ and showed that the separation between cluster and field stars is


around the mean value of the cluster to be M22 members, and

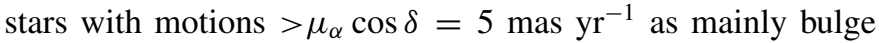
stars. In a vector-point diagram presented in Figure 2 we overlaid the vector measured here for the microlens on the vector for the bulge-M22 set from Chen et al. (2004). The microlens vector originates from the $(0,0)$ point, which refers to the cluster, and ends well within the bulge area. This confirms the geometry of the microlensing event with the source in the bulge and the lens in the globular cluster.

By fitting a model to the light curve Pietrukowicz et al. (2005) predicted that the lens is fainter than the source by $\sim 5 \mathrm{mag}$ in the $V$ band. At a distance $d_{\mathrm{M} 22}=3.2 \mathrm{kpc}$ and mean reddening $E(B-V)=0.38$ mag (Monaco et al. 2004) it is likely an M5 dwarf of an absolute brightness $M_{V} \sim 11.1 \mathrm{mag}$. Such star observed in the $K_{s}$ band would have $\sim 20.5 \mathrm{mag}$ (based on models from Brocato et al. 1998). The brightness of the faint object detected close to the target source in the $\mathrm{VLT} / \mathrm{NACO}$ image is $K_{s}=20.57 \pm 0.09 \mathrm{mag}$, which is in excellent agreement.

The VLT/NACO $K_{s}$-band image is the only available image containing both microlensing system components. We searched the HST archives for other high-resolution images. 


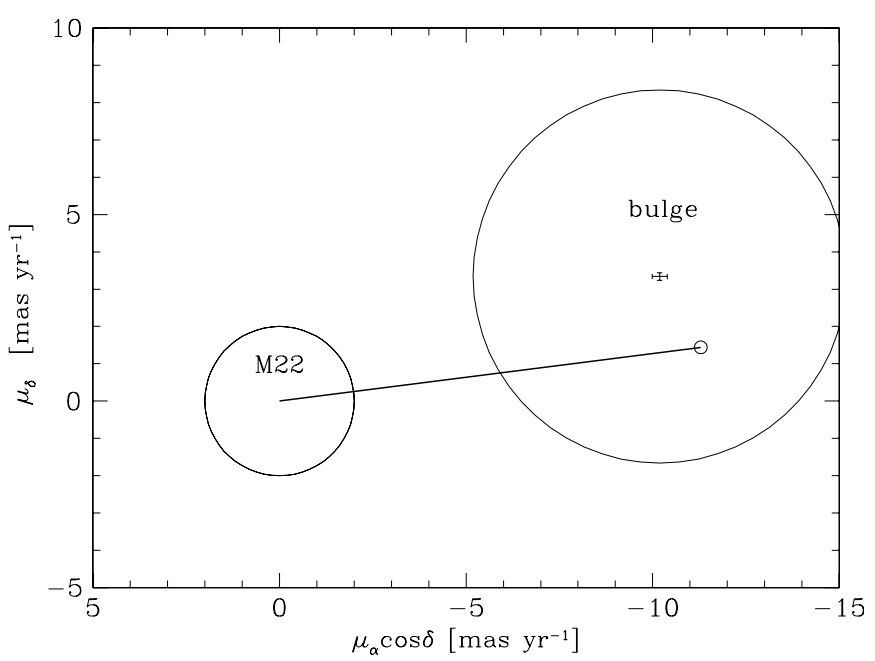

Figure 2. Vector-point diagram of relative proper motions in the $\mathbf{J} 2000$ equatorial coordinate system of the bulge with respect to the globular cluster M22 (based on Chen et al. 2004). Stars that would fall inside the circle of radius 2 mas yr$^{-1}$ centered at $(0,0)$ are considered to be cluster members, while stars that would fall inside the circle of radius 5 mas $\mathrm{yr}^{-1}$ centered at $(-10.19,3.34){\mathrm{mas} \mathrm{yr}^{-1}}^{-1}$ are very likely bulge stars. The relative motion between the microlens system components is shown as the solid line with the small circle representing the uncertainty. The length and direction of the vector unambiguously confirm that the source belongs to the bulge and the lens to the cluster.



Figure 3. OGLE-IV I-band light curve of the target object in years 2010-2011. Its constant brightness within $0.2 \mathrm{mag}$ confirms the microlesing nature of the event in 2000 July/August.

Unfortunately, in two HST/Advanced Camera for Surveys (ACS) images taken as a part of the GO 10775 program on 2006 Apr 1 our target lies 1".3 off the edge. The only HST image (jb1w01010, GO 11558) covering the M22 microlens was obtained on 2010 Mar 2 in the O [III] filter centered at $5023 \AA$ and with $\mathrm{FWHM}=86 \AA$. We checked that in this narrow-band filter all objects of similar brightness to the lens in the NACO image are below the detection limit. This supports the fact that the lens is a relatively red object.

We also checked brightness variations of the target object in recent OGLE data obtained with the $1.3 \mathrm{~m}$ Warsaw telescope at Las Campanas Observatory, Chile. The Optical Gravitational Lensing Experiment during its fourth phase (OGLE-IV), started in 2010 March, observes the globular cluster M22 occasionally once or twice a week. In Figure 3 we present the I-band light curve of the target object in years 2010-2011. The zero-point accuracy of the magnitude scale is about $0.1 \mathrm{mag}$. Constant brightness of the object within 0.2 mag corroborates that the episode of increasing brightness in 2000 July/August was a single event.

Theoretically we can estimate the probability of a chance configuration of two unrelated stars in the investigated area. We detected 342 stars in a brightness range $15.4<K_{s}<22.6$ mag in the $15^{\prime \prime} \times 15^{\prime \prime}$ field centered on the target source. Assuming Poisson statistics this gives a density of $1.52 \pm 0.08$ stars $\operatorname{arcsec}^{-2}$ or $0.074 \pm 0.004$ stars within 124.6 mas around the target. Eighty-two stars (corresponding to a fraction of 0.240 ),

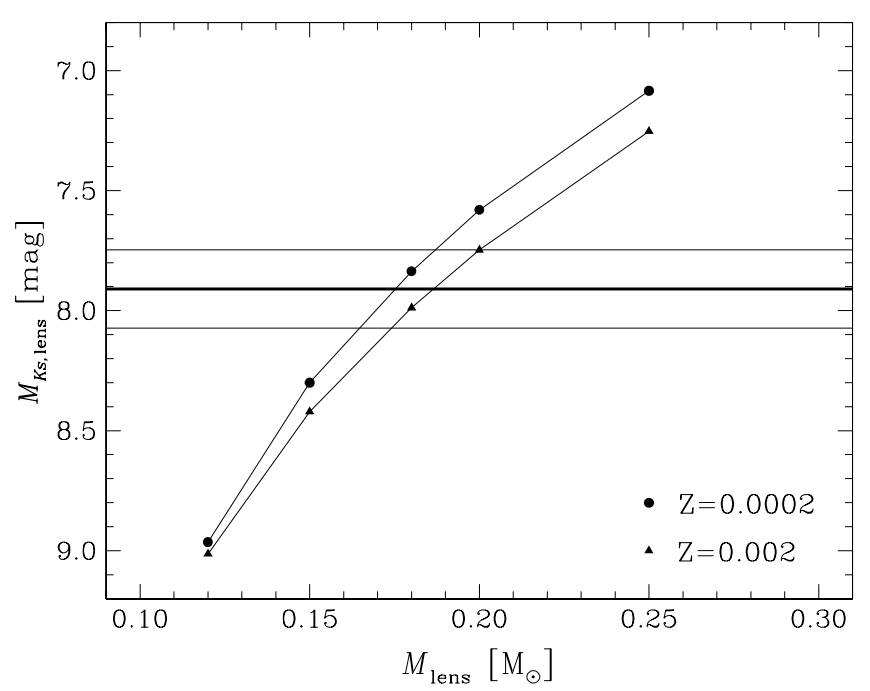

Figure 4. $K_{s}$-band absolute brightness for dwarfs of five different masses and two metallicities, $Z=0.0002$ and 0.002 , corresponding to $[\mathrm{Fe} / \mathrm{H}]=-2.00$ and -0.96 dex, respectively (data points taken from Brocato et al. 1998). The measured brightness of the lens and its uncertainty are marked with the horizontal lines.

being fainter than $K_{s}=20$ mag, could act as potential lenses in our case. The acceptable position angle of the lens ranges within \pm 38.5 off the M22-bulge relative proper motion direction, decreasing the chance by 0.214 . If we take into account all above requirements we find a $0.38 \% \pm 0.02 \%$ chance of such configuration at any location in the field. However, the observed position and brightness of both lens and source being in perfect agreement with the expectations unambiguously confirm the microlensing nature and geometry of the event detected in 2000.

\section{MASSES AND DISTANCES TO THE MICROLENS COMPONENTS}

The observed $K_{s}$-band brightness of the lensing star and the fact that it is located in the globular cluster M22 allows us to determine its type. According to Monaco et al. (2004) M22 lies at a distance $d_{\mathrm{M} 22}=3.2 \pm 0.2 \mathrm{kpc}$ from the Sun and has an average metallicity $[\mathrm{Fe} / \mathrm{H}]_{\mathrm{CG}}=-1.68 \pm 0.15$ dex in Carretta $\&$ Gratton (1997) scale. Reddening in the direction of M22 is spread between $E(B-V)=0.34$ and 0.42 mag (Richter et al. 1999). Using Rieke \& Lebofsky (1985) relations on absorption, where $A_{K}=0.112 A_{V}$, and where $A_{V}=3.1 E(B-V)$, we find $0.118<A_{K}<0.146 \mathrm{mag}$ for objects in M22. From this we obtain the absolute brightness of the lens $M_{K s}=$ $7.91 \pm 0.16 \mathrm{mag}$. Based on models from Brocato et al. (1998) we find the mass of the $\operatorname{star} M_{\text {lens }}=0.18 \pm 0.01 M_{\odot}$ (see Figure 4).

Knowing the distance to the lensing object $d_{\text {lens }}$, its mass $M_{\text {lens }}$, relative proper motion $\mu_{\text {rel }}$ between the source and lens, and timescale of the event $t_{\mathrm{E}}$ we can estimate distance to the source from the following relation:

$$
d_{\text {source }}=d_{\text {lens }}\left(1-\frac{c^{2} \mu_{\text {rel }}^{2} t_{\mathrm{E}}^{2} d_{\text {lens }}}{4 G M_{\text {lens }}}\right)^{-1},
$$

where $G$ is the gravity constant and $c$ the speed of light. The quantities $\mu_{\text {rel }}$ and $t_{\mathrm{E}}$ should be given in either heliocentric or geocentric frame. For the microlens in M22 we obtain $d_{\text {source }}=6.0 \pm 1.5 \mathrm{kpc}$ which places the source in the Galactic bulge, as expected from the relative motion. The large errors reflect mainly the uncertainty in the estimated duration of the microlensing event. 


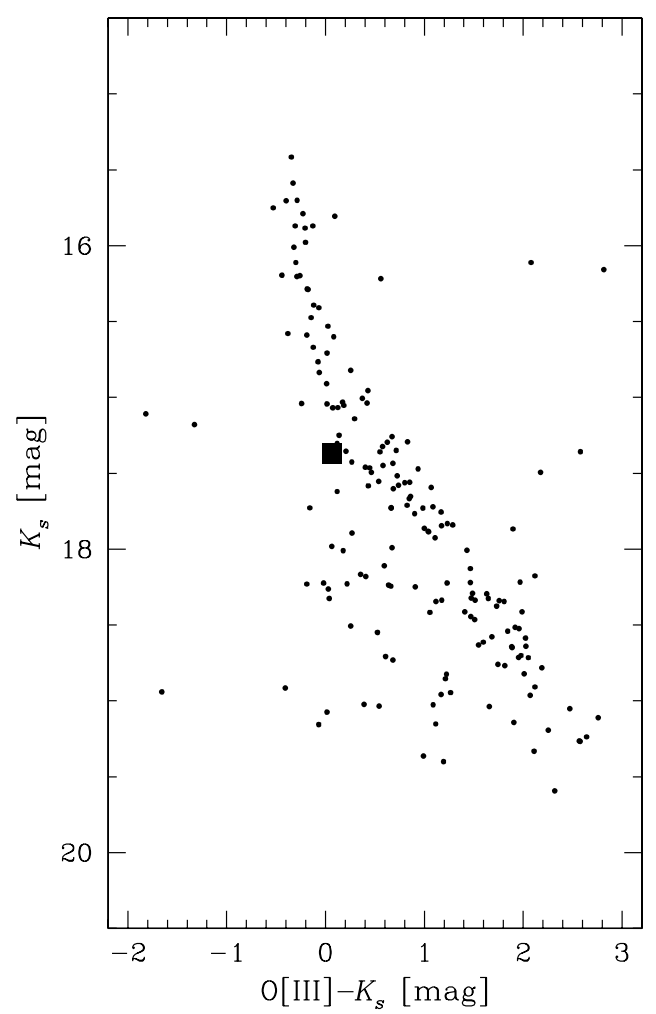

Figure 5. $K_{s}$ vs. O [III] $-K_{s}$ diagram for 175 stars present in both VLT/NACO and $H S T /$ ACS images in the M22-microlens area. The majority of the objects are main-sequence (MS) stars of the cluster. Location of the more distant source (marked with the filled square) shows that it is fainter than the M22 MS and consistent with a bulge MS star.

According to Schlegel et al. (1998) the total reddening in the cluster direction amounts to $E(B-V)=0.33$ mag. That implies that any stars located in the cluster field cannot be significantly more reddened than the cluster itself. If we assume the same absorption for the source located at $6.0 \mathrm{kpc}$ as for the cluster, $A_{K}=0.13 \mathrm{mag}$, from the observed brightness of the source $K_{s}=17.37 \mathrm{mag}$ we find it to be a solar-type star (Pietrinferni et al. 2006). The location of this object in a $K_{s}$ versus O [III] $-K_{s}$ diagram shown in Figure 5 supports this conclusion.

\section{SUMMARY}

In this Letter, we have shown that the microlensing event that occurred 2!33 from the center of the globular cluster M22 in $2000 \mathrm{July} /$ August involved a $0.18 \pm 0.01 M_{\odot}$ dwarf of the cluster and a background solar-like star located in the Galactic bulge. Almost 11 years after the event, using high-resolution near-IR image we resolved the two microlensing components.
The observed position of the source and lens stars as well as their brightness are consistent with the proposed earlier geometry of the event. Additional evidence comes from the constant brightness of the target object in the last two years (2010-2011).

We thank M. Jaroszyński for helpful discussions and A. Gould for drawing our attention to an inconsistency in our original calculation of the source distance. P.P. and A.U. are supported by funding to the OGLE project from the European Research Council under the European Community's Seventh Framework Programme (FP7/2007-2013)/ERC grant agreement No. 246678. P.P. is also supported by grant No. IP2010 031570 financed by the Polish Ministry of Sciences and Higher Education under Iuventus Plus programme. We gratefully acknowledge use of data from the ESO Public Survey programme ID 179.B-2002 taken with the VISTA telescope, and data products from the Cambridge Astronomical Survey Unit. D.M. and J.A.-G. are supported by Proyecto Fondecyt Regular 1090213, the BASAL Center for Astrophysics and Associated Technologies PFB-06, the FONDAP Center for Astrophysics 15010003, and the Milky Way Millennium Nucleus from the Ministry of Economia ICM grant P07-021-F.

\section{REFERENCES}

Alcock, C., Allsman, R. A., Alves, D. R., et al. 2000, ApJ, 542, 281 Alcock, C., Allsman, R. A., Alves, D. R., et al. 2001, Nature, 414, 617 Anderson, J., Cool, A. M., \& King, I. R. 2003, ApJ, 597, L137 Bond, I. A., Abe, F., Dodd, R. J., et al. 2001, MNRAS, 327, 868 Brocato, E., Cassisi, S., \& Castellani, V. 1998, MNRAS, 295, 711 Carretta, E., \& Gratton, R. G. 1997, A\&AS, 121, 95

Chen, D., Chen, L., \& Wang, J.-J. 2004, Chin. Phys. Lett., 21, 1673

de Luca, F., \& Jetzer, Ph. 2008, Int. J. Mod. Phys. D, 17, 2305

Harris, W. E. 1996, AJ, 112, 1487

Jetzer, Ph., Strässle, M., \& Wandeler, U. 1998, A\&A, 336, 411

Kaluzny, J., Thompson, I. B., Krzemiński, W., et al. 2005, in AIP Conf.

Proc. 752, Stellar Astrophysics with the World's Largest Telescopes: First International Workshop on Stellar Astrophysics with the World's Largest Telescopes, ed. J. Mikołajewska \& A. Olech (Melville, NY: AIP), 70 Kozłowski, S., Woźniak, P. R., Mao, S., \& Wood, A. 2007, ApJ, 671, 420 Minniti, D., Lucas, P. W., Emerson, J., et al. 2010, New Astron., 15, 433 Monaco, L., Pancino, E., Ferraro, F. R., \& Bellazzini, M. 2004, MNRAS, 349, 1278

Paczyński, B. 1994, Acta Astron., 44, 235

Pietrinferni, A., Cassisi, S., Salaris, M., \& Castelli, F. 2006, ApJ, 642, 797

Pietrukowicz, P., Kaluzny, J., Thompson, I. B., et al. 2005, Acta Astron., 55, 261

Richter, P., Hilker, M., \& Richtler, T. 1999, A\&A, 350, 476

Rieke, G. H., \& Lebofsky, M. J. 1985, ApJ, 288, 618

Sahu, K. C., Anderson, J., \& King, I. R. 2002, ApJ, 565, L21

Sahu, K. C., Casertano, S., Livio, M., et al. 2001, Nature, 411, 1022

Schlegel, D. J., Finkbeiner, D. P., \& Davis, M. 1998, ApJ, 500, 525

Stetson, P. B. 1987, PASP, 99, 191

Udalski, A., Żebruń, K., Szymański, M., et al. 2000, Acta Astron., 50, 1 